\title{
Design of Intelligent DC boost power supply for Pulse RADAR
}

\author{
Weiqing Zhang1, a, Ming Xia1, b \\ Ordnance Military School of Wuhan \\ Wuhan, China \\ a362493371@qq.com, bming@163.com
}

\begin{abstract}
Key Words: Pulse Radar, High voltage system, DC boost, Over current intelligent, protection Rectifier-Filter

Abstract.Design of DC boost circuit is very important for the reliable application of the high voltage part in pulse radar. A kind of DC power supply circuit based on resistance, capacitance and voltage regulator is presented in view of the requirement of the power supply voltage of the high voltage power system of a certain type of pulse radar. The hardware components, working principle of the circuit and especially the intelligent protection circuit for over current are analyzed. The design meets the requirements for the $350 \mathrm{~V}$ voltage of the high voltage system on the phase of power output. The $24 \mathrm{~V}-350 \mathrm{~V}$ DC boost power supply meets the requirements of the high voltage system fully,and all the design circuit works stably meets the requirements of the high voltage system fully,and all the design circuit works stably and reliably after verification. Intelligent protection circuit can protect the stability of high voltage pulse radar system effectively because of the characteristics of strong anti-interference ability, high sensitivity and high intelligence.
\end{abstract}

\section{Introduction}

Pulse radar technology is widely used in the information of ground defense system with its rapid development.However, the large power transmitter power supply which has been changed to various functional modules is removed in the current pulse radar. So,the number of secondary power in radar increased significantly which makes power system being more and more complex with the failure rate increasing significantly. Common faults such as over voltage, under voltage, over current, short circuit and so on, are often difficult to avoid,because radar is often working in complex electromagnetic environment. Therefore, it is the key to ensure the safe operation of the power system, which is the fast location of the fault, the protection of the power supply and the fault alarm.

\section{Hardware component}

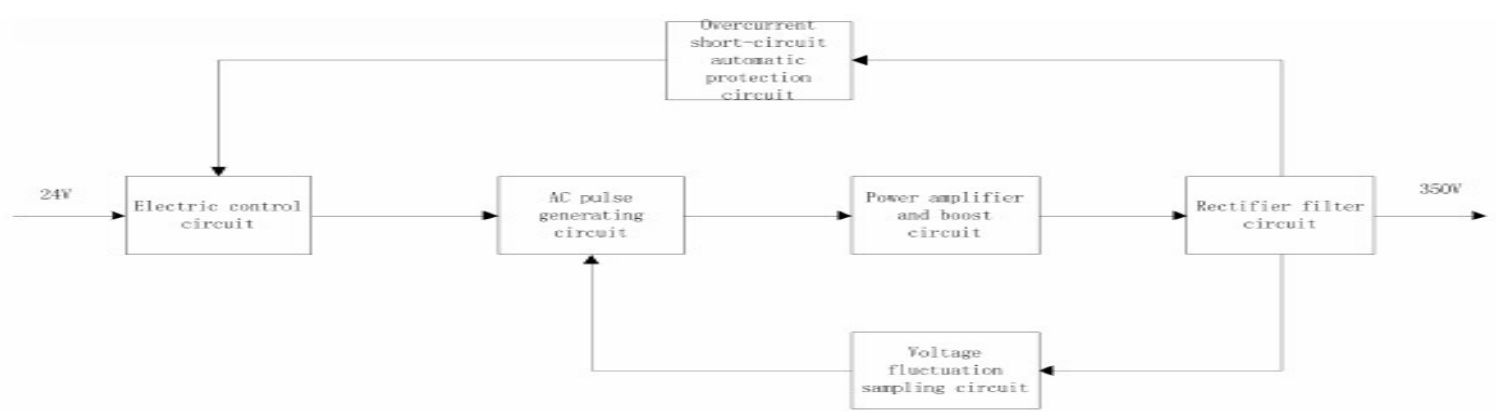

Fig. $124 V-350 V$ DC boost circuit block diagram

In this paper, we design a power supply module which will boost the DC $24 \mathrm{~V}$ to $350 \mathrm{~V}$ according to the requirement for the $350 \mathrm{~V}$ in high voltage system. This circuit has many functions such as 
DC-AC-DC converter, boost, voltage regulator, over current protection, power control and so on. It is composed of the electric control circuit, the pulse generating circuit, the power amplifier and booster circuits, the rectifier filter circuit, the over current short circuit automatic protection circuit, the voltage fluctuation sampling circuit in the hardware. The composition is shown in figure 1: composed of the transistor V2 (3 dk104a), three-terminal voltage regulator D3 (2 cw7812 voltage $12 \mathrm{v}$ ), resistance R14, R13, capacitor C11, C13, C14, C28. The pulse generating circuit which can generate the pulse signal with the frequency $22.5 \mathrm{kHz}$, the period $44.4 \mathrm{~s}$ and the pulse width of $19.5 \mathrm{~s}$, is composed of pulse width modulator D1(UC1526/883 b), zener diode V5 and V6, V7 and V8 (2 cw5245 15 v) voltage regulator, transformer T1, resistance R6, R9, R12, R15, R16 and R17, $\mathrm{R} 18$, capacitor C1, C4, C5, C6, C7, C8, C9. The power amplifier and booster circuits is composed of the field effect switch tube V15, V16 rf15d (1), diode V17, V18 (MUR090E), transient voltage suppression diodes V27, V28 (TVP1525), transformer T4, resistance R27, the R28, R29, R30, capacitance C18, C19, C20, of C21, C26.The rectifier filter circuit is composed of the diode V19, V21, V24, V26 (MUR090E), filtering inductance L1, L2, L3, filter capacitance C22, C23, C24, C25 resistance of $\mathrm{R} 31$.

The over current short circuit automatic protection circuit is composed of over current sampling inductance T3, monostable trigger D2 (SG54121J), triode V4, V3 (3 g33a), voltage regulator tube V9 cw5241 (2), rectifier diode V11, V13, V14 (IN4140), filter capacitor C10, C12, C15, C16, C17, potentiometer RP3, resistance R10, R11, R19, R20, R21, R22, R23, R25, R26.The voltage fluctuation sampling circuit which can carry on the sampling to the voltage fluctuation, the realization of feedback to control the voltage fluctuation, is composed of the potentiometer RP1, resistor R1, R2, R3 and R4, R7, capacitance C2 and C3.

Working principle

The electric control circuit which can carry out electric control to the input 24 voltage, is Voltage regulation principle. The sampling voltage is taken out from the center head of the sampling circuit RP1 for the voltage fluctuation when the output DC voltage amplitude of $350 \mathrm{~V}$ has a fluctuation and is greater than $0.5 \mathrm{~V}$. Then, this sampling voltage is sent to D1 through R7、C2、R4、C3, which can change the conduction time of V15 and V16 by changing the pulse width of the oscillation signal. At last, the output voltage can be obtained with the range of $350 \mathrm{~V} \pm 0.5 \mathrm{~V}$ stably. DC booster principle. The DC $24 \mathrm{~V}$ power will be divided into two channels after getting into the power supply circuit. One signal will be sent to the source pole of V15, V16 through the T3 and T4, the other will be sent to the electric control circuit V2.

The pulse width modulator D1 generates the pulse signal with the frequency $22.5 \mathrm{kHz}$, the period $44.4 \mathrm{~s}$ and the pulse width of $19.5 \mathrm{~s}$ in the use of $12 \mathrm{v}$ output voltage from the pin 2 of three-terminal voltage regulator D3, which is generated by the $24 \mathrm{v}$ power supply in the pin 1 which come from the set-emitter of V2 through the base of electric control circuit V2 after the dividing pressure of R14, R13.This signal generates coupling two mutual difference of V6、V7、V8 and make the V15、V16 push-pull conduction, cut-off after presenting effect switch tube between gate and drain of them respectively, through transformer T1 after outputting from pin 13,16 of $\mathrm{D} 1$. Then the $350 \mathrm{~V} \pm 0.5 \mathrm{~V}$ dc voltage will be output stability after the pulsating voltage which is formed in primary of T4 going through the T4 booster, V19, V21, V24, V26 bridge rectifier and L1, C22, L2, C23, L3, C24, C25 triple filter. At last, the output will be divided into two channels, one of which is the output, the other one of which is sent to sampling circuit R1, R2, R3 and RP1 for voltage fluctuation whose normal sampling voltage $5 \mathrm{v}$ which is taken out by the center head of the RP1 makes the circuit after the D1 work normally through the R7, C2, R4, C3, C20, V17, C21, V18 is not only used to suppress the high frequency interference, but also can be used in forming a loop for the self-induction electromotive force of sudden jump which is created by the T4 primary when the V15, V16 cut-off suddenly, in order to avoid the electromotive force and $24 \mathrm{v}$ external power superposition and burn out the field effect tube V15, V16. While the R29, R30 are used to put off the voltage stored on C21, C20 when power off.V27, V28 have the function of bidirectional voltage surge suppression, which can protect the field effect tube V15, V16, etc, by being used in ac pulse shaping, limiting voltage. While, the V5 and V6, V7 and V8 can carry out the protection for the 
field effect tube V15, V16 from the gate and drain after being in reverse concatenated, which is different from the V27, V28. In normal operation, there is no situation of over current and over voltage in the $350 \mathrm{~V}$ power supply and no signal $(<0.3 \mathrm{~V})$ output in secondary pole of over-current sampling coil T3, so, there is no voltage in base of over-current automatic protection circuit V4, that makes the collector have high level $(5 \mathrm{~V})$, which is sent to the inverse pin 3,4 of single steady state trigger D2.Then,the $350 \mathrm{~V}$ power output normal because of pulse width modulator D1 normal operation which is made by the high level $(5 \mathrm{~V})$ which is sent to D1 from the Q inverting output 1 pin. The high level $(11 \mathrm{~V})$ which came from collector of V3 which is cut-off because of the partial voltage of low level $(0.3 \mathrm{~V})$ from Q output pin 6 through R20, R19, is the regulator of V9 is sent to the "350V" indicator that is positive for the light emitting diode, and the indicator light is on, indicating that the out of this power supply module is normal $350 \mathrm{~V}$.

\section{Automatic intelligent protection principle.}

The secondary of T3 will output the pulse induction signal $(>12 \mathrm{~V})$ of over-current rapidly because of the sudden over-current of $350 \mathrm{~V}$ power supply due to the failure of the external load circuit, or the power supply circuit fault. The power and its external load will be protected because the circuit has been stopped working without $350 \mathrm{~V}$ power output, that is realized through making the pulse width modulator D1 stop oscillation by the low level $(0.2 \mathrm{~V})$ came from 1 pin of Q inverting output which is input from inverting input 3 and 4 pin of single steady state trigger D2 which is the low level signal output from the collector of the transistor V4 whose conduction is caused of the pulse induction signal which is sent to the base of V4 through RP3、V11、R23 after V13, V14 rectifier and C15, C16 filtering. While, the "350V" indicator light goes out because of the low level came from the collector of $\mathrm{V} 3$ after the conduction because of the partial voltage of high level $(>3.5 \mathrm{~V})$ on the base of V3 through R19、R20,that can indicate that the power supply circuit is fault without $350 \mathrm{~V}$ power output.

\section{Conclusion}

In this paper, a simple and practical power supply of $350 \mathrm{~V}$ power which can realize the function of DC boost, voltage ripple suppression, over current automatic protection and so on has been designed with the development of radar power supply system,on the basic of analysis of main functions, hardware components, working principle and the characteristics of intelligent protection circuit. The practical application shows that the protection circuit is stable and reliable, with high sensitivity, and can be used to carry out the alarm and protect the boost module accurately. The false alarm rate is below $2 \%$, the fault alarm rate greater than $98.5 \%$, the fault isolation rate greater than $95 \%$, all of which meets the requirements of the protection for high voltage power supply.It provides the guarantee for improving the working performance of the high voltage system in pulse radar and the whole radar system to work more safely and reliably.

\section{References}

[1]Yujie Fang, Binghua Su, Lingxia Hang. Study on ripple rejection of switching power supply[J]. Modern electronic technology, 2012 (5): 37-39.

[2] Lu Qiusheng. Bus technology used in digital power[J].Electronic test, 3509(9): 35-37.

[3] JIA Kai, ZHENG Chunhui, CHEN Weilian. Design and implementation of dual power supply circuit based on TMS320F2812[J]. ELECTRONIC ENGINEER, 3508(9): 21-23.

[4] Chen Lin Li Shuqin Lin Hui. Digital Power Management and Power Management Bus[J].

Electric drive, 3508(8): 19-21.

[5] ZHANG Guang-xin, YANG Geng. Power Supply Design for Controller of Active Power Filter[J]. Power Electronics, 2010(2): 25-27. 